\title{
Rotational sound in disordered granular materials
}

\author{
Kuniyasu Saitoh, ${ }^{1,2, *}$ Rohit K. Shrivastava, ${ }^{3}$ and Stefan Luding ${ }^{3}$ \\ ${ }^{1}$ Research Alliance Center for Mathematical Sciences, Tohoku University, 2-1-1 Katahira, Aoba-ku, Sendai 980-8577, Japan \\ ${ }^{2}$ WPI-Advanced Institute for Materials Research, Tohoku University, 2-1-1 Katahira, Aoba-ku, Sendai 980-8577, Japan \\ ${ }^{3}$ Faculty of Engineering Technology, MESA+, University of Twente, P.O. Box 217, 7500 AE Enschede, The Netherlands
}

(Received 28 September 2018; published 24 January 2019)

\begin{abstract}
We numerically study the evolution of elastic standing waves in disordered disk systems with a focus on the dispersion relations of rotational sound. As on a lattice, the rotational mode exhibits an optical-like dispersion relation in the high frequency regime, representing a shoulder in the vibrational density of states and fast oscillations of the autocorrelations of rotational velocities. If tangential stiffness between the disks is large enough, a lattice-based model perfectly describes the dispersion relation of the rotational mode. If it is comparable to or smaller than the normal stiffness, the model fails for short wavelengths. However, the dispersion relation then follows the model prediction for the transverse mode, implying that the fast oscillations of disks' rotations switch to acousticlike behavior. We evidence such a transition from rotational to transverse modes by analyzing their respective participation of different degrees of freedom to the eigenvectors.
\end{abstract}

DOI: 10.1103/PhysRevE.99.012906

\section{INTRODUCTION}

Internal degrees of freedom bring about rich physical phenomena where micropolar rotations of grains in granular materials are one of the typical and classical examples. An important feature of granular material is its sound characteristics [1] as crucial to oil and gas exploration, geotechnical investigations of soil, and understanding of seismic waves and earthquakes [2]. To better interpret measurements, a model which incorporates the microstructure is required. Incorporating the rotational degrees of freedom for the internal microstructure has been the basis of the continuum theory of Cosserat and Cosserat [3]. So far, one of the most striking aspects of Cosserat behavior is the existence of rotational sound [4]. This has been extensively studied by experiments [5] and numerical simulations [6-8] of granular crystals where the characteristic "optical-like" dispersion relations, beyond Cosserat, are well predicted by the theory of particles on a lattice $[9,10]$.

Despite these successes of lattice theory, granular materials in nature are mostly disordered $[11,12]$. Small deviations from the lattice display the characteristic low frequency behavior [13]. In addition, various anomalies in acoustic sound in disordered media, e.g., small dips in phase speeds and deviations from the theory of Rayleigh scattering, have recently been clarified by experiments on amorphous solids [14,15] and numerical simulations of randomly arranged particles [16-19]. Anomalies in the vibrational density of states (vDOS), e.g., the boson peak near the glass transition temperature [20-26] and a characteristic plateau near the jamming transition [27-30], are not predicted by the classical theory of elasticity. Moreover, shock waves [31-34] and solitary

*kuniyasu.saitoh.c6@tohoku.ac.jp wave propagation [35,36] are interesting properties of real disordered systems, although they are due to nonlinearity of the interaction forces and anharmonic vibrations. Neglecting the latter phenomena and reducing to rather simple twodimensional (2D) model systems, we focus on the question: How does configurational disorder alter the characteristics of rotational sound?

In this paper, we numerically investigate sound in twodimensional disordered disk systems where tangential elastic forces between the disks enable the rotational sound. Since we are interested mostly in elastic waves and want to apply methods that require that the contact network does not change, we restrict ourselves to the case of very small amplitudes of perturbations, which has as a consequence that the frictional Coulomb sliding limit is never reached by the contacts. Molecular dynamics (MD) type simulations, for which these are no limitations, show similar results as long as the amplitude of waves is not too large so that only a few sliding and/or opening or closing events happen without larger scale structural rearrangements. First, we analyze eigenfrequencies of the systems and then simulate the evolution of elastic standing waves of longitudinal (L), transverse (TR), and rotational (RT) modes. Calculating autocorrelation functions and power spectra of these modes, we examine the dependence of their dispersion relations on the strength of tangential forces. We describe optical-like dispersion relations of the RT mode by a modified lattice-based model. Interestingly, we find that the RT mode exhibits a remarkable transition from the optical-like branch to an acoustic branch at a characteristic wavelength. We analyze closer these transitions by eigenvectors of the disk system. In the following, we explain our numerical methods in Sec. II and show our results in Sec. III. We discuss our results in Sec. IV and conclude our findings in Sec. V. We summarize full details of our methods in Appendices $\mathrm{A}-\mathrm{C}$ and provide additional data in Appendices D-G. 


\section{METHOD}

To study sound in disk systems, we introduce their dynamical matrix. If the system consisting of $N$ disks is initially in mechanical equilibrium, small vibrations of the disks around their initial positions $\left\{\mathbf{r}_{i}(0)\right\}(i=1, \ldots, N)$ are described by the equations of motion,

$$
\mathcal{M} \ddot{\mathbf{u}}(t)=-\mathcal{D} \mathbf{u}(t),
$$

where $t$ denotes time and the $3 N$-dimensional displacement vector,

$$
\mathbf{u}(t) \equiv\left[\mathbf{u}_{1}(t), \theta_{1}(t), \ldots, \mathbf{u}_{N}(t), \theta_{N}(t)\right]^{\mathrm{T}}
$$

includes translational displacements on the $x y$ plane, $\mathbf{u}_{i}(t) \equiv$ $\mathbf{r}_{i}(t)-\mathbf{r}_{i}(0)$, and angular displacements $\theta_{i}(t)$. On the lefthand side of Eq. (1), the $3 N \times 3 N$ mass matrix $\mathcal{M}$ is diagonal. On the other hand, $\mathcal{D}$ is the $3 N \times 3 N$ Hessian which consists of second derivatives of elastic energy (see Appendix A for full details). In this paper, we model the elastic energy by harmonic potentials in normal and tangential directions where the normal (tangential) stiffness is given by $k_{n}\left(k_{t}\right)$. We prepare initial disordered configurations $\left\{\mathbf{r}_{i}(0)\right\}$ by MD simulations where every disk has the same mass $m$ (see Appendix B). In the following analyses, we scale every length and time by the mean disk diameter $d_{0}$ and time unit $t_{0} \equiv \sqrt{m / k_{n}}$, respectively.

\section{RESULTS}

In this section, we show our numerical results of the vDOS (Sec. III A) and dispersion relations (Sec. III B). We compare the dispersion relations with the lattice-based model (Sec. III C) and analyze the transition behavior of the RT mode (Sec. IIID). Then, we discuss the frequency range of the RT mode (Sec. III E).

\section{A. Vibrational density of states}

Assuming vibrational motions of the disks around initial positions, we substitute $\mathbf{u}(t)=\overline{\mathbf{u}} e^{I \omega t}$ into Eq. (1), where $\overline{\mathbf{u}}, I$, and $\omega$ are the amplitude, imaginary unit, and angular frequency, respectively. Then, we numerically solve an eigenvalue problem $\mathcal{M}^{-1} \mathcal{D} \overline{\mathbf{u}}_{q}=\omega_{q}^{2} \overline{\mathbf{u}}_{q}$ to find the eigenfrequencies $\omega_{q}$ and eigenvectors $\overline{\mathbf{u}}_{q}(q=1, \ldots, 3 N)$. Figure 1 displays distributions of the eigenfrequencies, i.e., vDOS (solid lines) where we averaged every vDOS over 100 different initial configurations of $N=2048$ disks. Here, we change the stiffness ratio,

$$
\rho \equiv \frac{k_{t}}{k_{n}}
$$

and also plot the result of frictionless disks, i.e., $\rho=0$ (dotted line). The highest peak around $\omega t_{0} \simeq 2.3$ for frictionless disks shifts to higher frequencies with increasing the stiffness ratio. In addition, a shoulder, which does not exist in the vDOS of frictionless disks, develops for higher frequencies $(2.3 \lesssim$ $\left.\omega t_{0} \lesssim 6.6\right)$ such that the cutoff frequency $\omega_{c}$ at the right end of vDOS, i.e., $g\left(\omega>\omega_{c}\right)=0$, greatly increases from $2.3 t_{0}^{-1}$ to $6.6 t_{0}^{-1}$. Therefore, the high frequency band (the shaded region in Fig. 1) is characteristic of disordered disk systems with tangential elastic forces. Note that there is a secondary peak

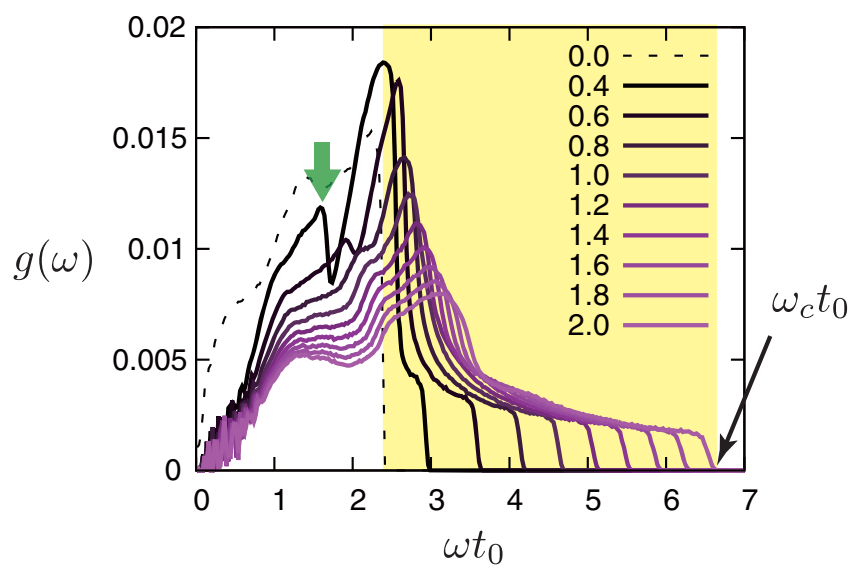

FIG. 1. The vDOS of disordered disk systems with tangential elastic forces (solid lines) where the area fraction is $\phi=0.9$ and the stiffness ratio $\rho$ increases as listed in the legend. The dotted line is the vDOS of disordered frictionless disks with the same area fraction. The shaded region represents the high frequency band for $\rho=2$ where the vDOS ends at the cutoff frequency $\omega_{c}$ as indicated by the arrow. The green vertical arrow indicates a secondary peak of vDOS for $\rho=0.4$.

if the stiffness ratio is small and the overpopulation of low frequency states for $\rho=0$ vanishes and becomes linear within the fluctuations for $\rho>0$. In Appendix D, we show that the vDOS in the high frequency regime is insensitive to the area fraction of the disks $\phi$. Thus, we use $\phi=0.9$ in the following analyses.

\section{B. Dispersion relations}

To investigate how elastic waves evolve in the systems, we employ a similar method as Gelin et al. [16]: We numerically integrate the equations of motion Eq. (1) under periodic boundary conditions. The number of disks is now increased to $N=32768$, and initial velocities of the disks are given by sinusoidal standing waves,

$$
\left\{\dot{\mathbf{u}}_{i}(0), \dot{\theta}_{i}(0)\right\}=\left\{\mathbf{A}, A_{\theta}\right\} \sin \left[\mathbf{k} \cdot \mathbf{r}_{i}(0)\right],
$$

where $\mathbf{A}$ and $A_{\theta}$ are small amplitudes and $\mathbf{k}$ is the wave vector. Combining different amplitudes and wave vectors, we activate three different elastic waves, i.e., L, TR, and RT modes as listed in Table I [37]. The RT mode represents the evolution of micropolar rotations [6] which do not exist in frictionless systems. Because Eq. (1) describes purely harmonic

TABLE I. The L, TR, and RT modes excited by different combinations of $\mathbf{A}=\left(A_{x}, A_{y}\right), A_{\theta}$, and $\mathbf{k}=\left(k_{x}, k_{y}\right)$, where $\mathbf{A}$ and $A_{\theta}$ are scaled by $d_{0} / t_{0}$ and $t_{0}^{-1}$, respectively.

\begin{tabular}{cccccc}
\hline \hline & $A_{x}$ & $A_{y}$ & $A_{\theta}$ & $k_{x}$ & $k_{y}$ \\
\hline L & $10^{-3}$ & 0 & 0 & $k$ & 0 \\
& 0 & $10^{-3}$ & 0 & 0 & $k$ \\
TR & $10^{-3}$ & 0 & 0 & 0 & $k$ \\
& 0 & $10^{-3}$ & 0 & $k$ & 0 \\
RT & 0 & 0 & $10^{-3}$ & $k$ & 0 \\
& 0 & 0 & $10^{-3}$ & 0 & $k$ \\
& 0 & & & &
\end{tabular}



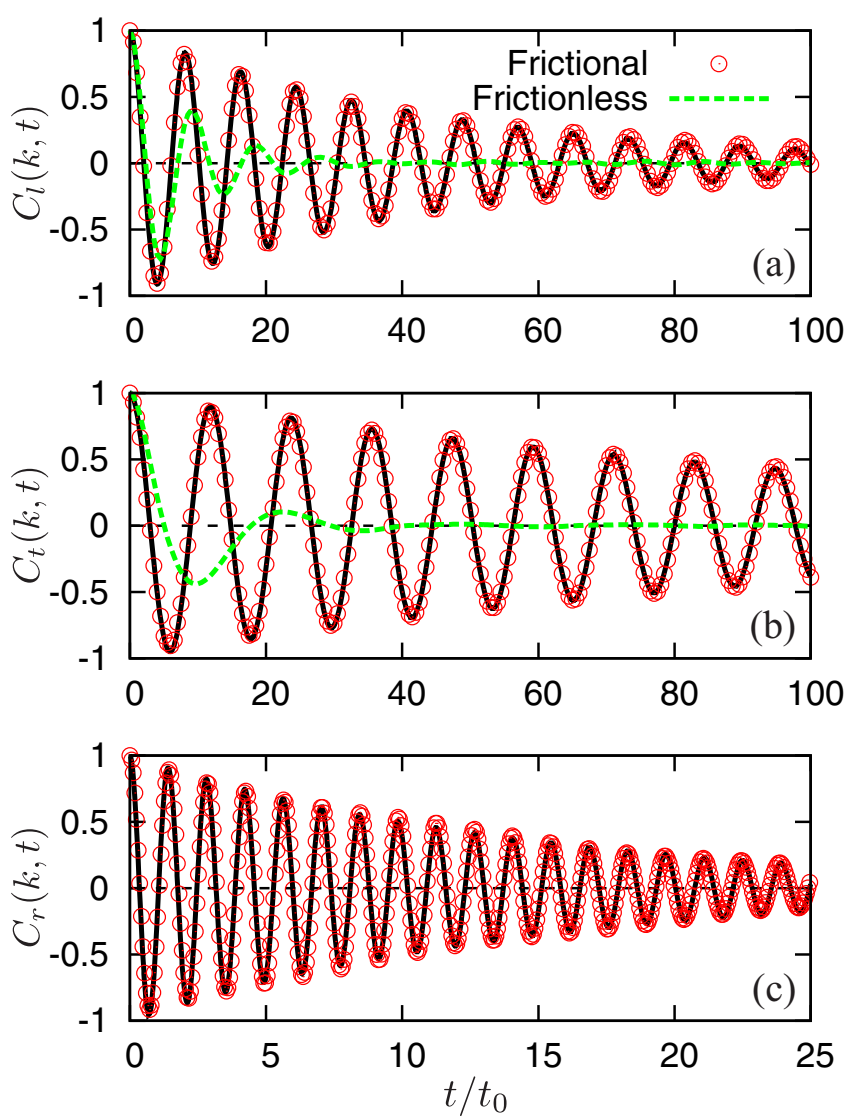

FIG. 2. The VAFs of (a) L, (b) TR, and (c) RT velocities, where $\rho=1$ and $k d_{0} \simeq 0.38 \pi$ are used. The open circles are obtained from numerical solutions of Eq. (1), whereas the solid lines represent damped oscillations Eq. (5). In (a) and (b), our results of frictionless disks $\rho=0$ are also shown (green dotted lines). Note the different horizontal scale in (c).

oscillations of the disks around initial positions, any anharmonic behavior, e.g., opening and closing contacts [38], is not taken into account.

From numerical solutions of Eq. (1), we calculate normalized velocity autocorrelation functions (VAFs) of L, TR, and RT velocities as $C_{l}(k, t), C_{t}(k, t)$, and $C_{r}(k, t)$, respectively, for various wave numbers $k \equiv|\mathbf{k}|$ in Eq. (4) (see Appendix C). Figure 2 shows the time development of the VAFs (open circles) where we also plot our results of frictionless disks [green dotted lines in (a) and (b)]. As expected, the oscillations of the $\mathrm{L}$ mode are faster than those of the TR mode [Figs. 2(a) and 2(b)]. The oscillations of the $L$ and TR modes are faster in the disk systems with tangential elastic forces that increase the macroscopic elastic constants. We also note that the decay of the VAFs, which is caused by scattering attenuation of the acoustic sound, becomes weaker. On the other hand, the oscillation of the RT mode is much faster than those of the acoustic modes [Fig. 2(c)], indicating that eigenmodes in the high frequency band (the shaded region in Fig. 1) are closely related to micropolar rotations of the disks. Moreover, the VAF of the RT mode decays much faster, implying a stronger scattering attenuation of the rotational sound.

Dispersion relations of the L, TR, and RT modes are represented by their power spectra, i.e., $S_{l}(k, \omega), S_{t}(k, \omega)$, and
$S_{r}(k, \omega)$, respectively (Appendix C). Figure 3 displays logarithms of the spectra $\ln S_{\alpha}(k, \omega)(\alpha=l, t, r)$. In the dispersion relations of both the L and the TR modes [Figs. 3(a) and 3(b)], we observe strong ordinary acoustic branches where the speed defined as the slope $\lim _{k \rightarrow 0} \omega / k$ of the L mode is higher than that of the TR mode. On the other hand, the RT mode exhibits a characteristic optical-like branch [Fig. 3(c)] which exists only in a high frequency regime $\left(2.5 \leqslant \omega t_{0} \leqslant 4.7\right)$ as indicated by the double-headed vertical arrow in Fig. 3(c). Because the vDOS is given by an integral of the dispersion relation over the whole wave number range [1], the high frequency band in the vDOS (the shaded region in Fig. 1) is the result of the optical-like branch. In addition, the weak optical-like branch in Fig. 3(b) and the weak acoustic branch in Fig. 3(c) mean that rotations are always coupled with transverse (shear) motions [6].

\section{Lattice-based model}

The optical-like branch of the RT mode, which is spanning the high frequency band, is a striking feature of the disk systems involving rotational degrees of freedom. To quantitatively analyze its properties, we extract dispersion relations from numerical solutions of Eq. (1). For this purpose, we fit damped oscillations to the VAFs as

$$
C_{\alpha}(k, t)=e^{-\gamma_{\alpha}(k) t} \cos \omega_{\alpha}(k) t
$$

( $\alpha=l, t, r$ ). The dispersion relation of each mode is given by the dominant frequency $\omega_{\alpha}(k)$, whereas the scattering attenuation of each mode is quantified by the attenuation coefficient $\gamma_{\alpha}(k)$ [39]. The solid lines in Fig. 2 represent the damped oscillations Eq. (5) where we confirm perfect agreements with the VAFs by adjusting the parameters $\omega_{\alpha}(k)$ and $\gamma_{\alpha}(k)$. In Appendix E, we summarize our results of the acoustic modes: Small dips are observed in the phase speeds $c_{\alpha}(k) \equiv \omega_{\alpha}(k) / k$, whereas the attenuation coefficients obey the Rayleigh prediction of scattering attenuation $\gamma_{\alpha}(k) \sim k^{3}(\alpha=l, t)$, which is not the case for frictionless disks [16].

Figure 4 displays dispersion relations of the optical-like RT modes $\omega_{r}(k)$ (symbols) where the frequency band shifts to higher frequencies with increasing the stiffness ratio $\rho$. To explain such a dependence of optical-like branches on $\rho$, we modify the discrete model of granular crystals [6]. Assuming that the long wavelength behavior of elastic waves is not affected by the difference between microstructures (order and disorder), we employ the same functional forms of the dispersion relations derived for monodispersed grains on a lattice (see also Appendix F). Here, we represent effects of disorder by: (i) the decrease in sound speed due to dispersion and (ii) modifying the lattice constant. Then, the dispersion relations of the TR and RT modes are

$$
\begin{aligned}
& \omega_{t}(k)=a_{t} \sqrt{f(k, \rho)-\sqrt{g(k, \rho)}}, \\
& \omega_{r}(k)=a_{r} \sqrt{f(k, \rho)+\sqrt{g(k, \rho)}},
\end{aligned}
$$

respectively, where the prefactor $a_{\alpha}(\alpha=t, r)$ is the result of the decrease in sound speed and the functions are 

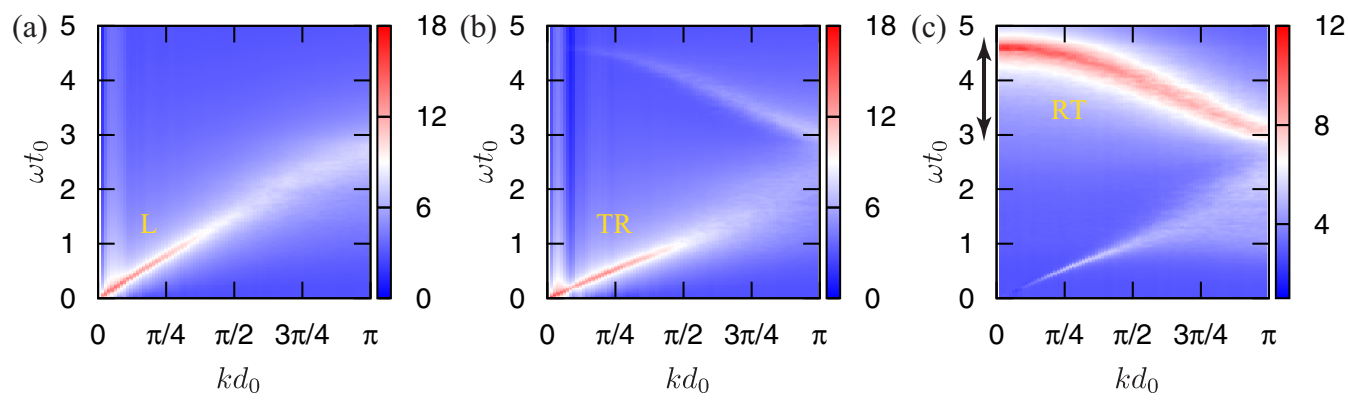

FIG. 3. Dispersion relations of the (a) L, (b) TR, and (c) RT modes, where $\rho=1$. The gray scale represents logarithms of the power spectra ln $S_{\alpha}(k, \omega)(\alpha=l, t, r)$ where the frequency and wave number are scaled by using $t_{0}$ and $d_{0}$, respectively. The frequency band of the RT mode is indicated by the double-headed vertical arrow in (c).

introduced as

$$
\begin{gathered}
f(k, \rho)=2 \sin ^{2}(k l)+9 \rho \cos ^{2}(k l)+11 \rho, \\
g(k, \rho)=4 \sin ^{4}(k l)+\rho(300 \rho-4) \cos ^{2}(k l) \\
-\rho\left(\frac{121}{4} \rho+21\right) \sin ^{2}(2 k l)+\rho(\rho+4) .
\end{gathered}
$$

In Eqs. (8) and (9), we estimate the length scale $l$ by considering the first Brillouin zone $|k l| \leqslant \pi / 2$ : Equating the maximum wave number $k_{\max } \equiv \pi / 2 l$, in the model with $\pi / d_{0}$, we obtain $l \approx d_{0} / 2$, where $d_{0}$ is the mean disk diameter. The lines in Fig. 4 are the model predictions of $\omega_{r}(k)$ [Eq. (7)], where $a_{r} \simeq 0.73 t_{0}^{-1}$ and $l \simeq 0.446 d_{0}\left(\approx d_{0} / 2\right)$ are used for all $\rho$. In this figure, all the dispersion relations for long wavelengths $k d_{0} \lesssim \pi / 2$ are consistent with the lattice-based model since the difference in microstructures, i.e., order and disorder, should not affect the long wave behavior (see Appendix F for the cases of L and TR modes). Moreover, if the stiffness ratio is fairly large $\rho>1$, the dispersion relations are perfectly described by the model for all wavelengths. However, if the stiffness ratio is small $\rho<1$, we observe deviations from the

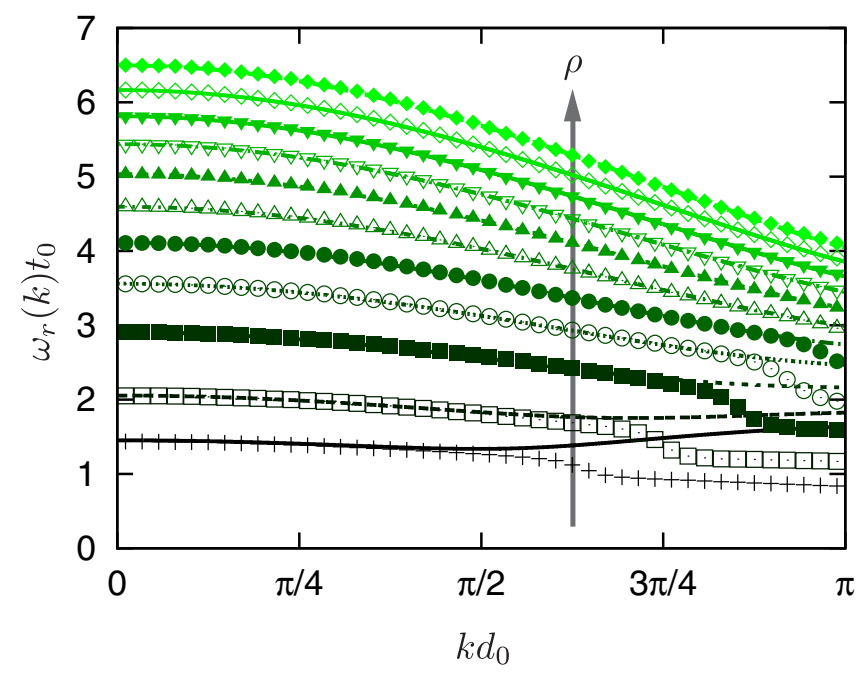

FIG. 4. Dispersion relations of the RT mode where the symbols are $\omega_{r}(k)$ in Eq. (5) and the lines are the model predictions Eq. (7). The stiffness ratio increases as $\rho=0.1,0.2,0.4,0.6,0.8,1.0,1.2$, $1.4,1.6,1.8$, and 2.0 (as indicated by the arrow). model for short wavelengths $k>k_{c}$ where the characteristic wave number $k_{c}$ monotonously decreases with decreasing stiffness ratio.

\section{Transition behavior of the RT mode}

To clarify the deviations in the short wavelengths, we closely look at the dispersion relations with small stiffness ratios. Figure 5 shows (a) the logarithm of the power spectrum $S_{r}(k, \omega)$ and (b) dispersion relations $\omega_{t}(k)$ and $\omega_{r}(k)$, obtained from the fitting to VAFs [Eq. (5)] where the stiffness ratio is given by $\rho=0.2$. In Fig. 5(a), the optical-like branch ends at $k=k_{c}$ and drops to another branch in $k>k_{c}$, which makes a small gap in the frequency between $1.2<\omega t_{0}<1.5$. Accordingly, in Fig. 5(b), the dispersion relation of the RT mode (squares) suddenly drops to lower values at $k=k_{c}$ [from branch (iv) to (ii)]. By contrast, that of the TR mode (crosses) jumps from branch (i) to (iii) such that the TR (RT) mode dominates higher (lower) frequencies in the short wavelengths. The dispersion relation of the RT mode in $k<k_{c}$ agrees with the model of $\omega_{r}(k)$ [Eq. (7), the solid line in Fig. 5(b)], whereas it is well explained by the model of $\omega_{t}(k)$ [Eq. (6), the dotted line in Fig. 5(b)] if $k>k_{c}$. Therefore, it seems that micropolar rotations exhibit a transition from the optical-like fast oscillations to the acoustic wave behavior.

We examine the transition behavior by the eigenvectors of the dynamical matrix $\overline{\mathbf{u}}_{q}=\left\{\bar{u}_{i x}, \bar{u}_{i y}, \bar{\theta}_{i}\right\}$, i.e., displacements associated with each eigenfrequency. We quantify kinetic energy for each degree of freedom by

$$
\begin{aligned}
K_{v} & \equiv \sum_{i} \frac{m}{2 N} \dot{\bar{u}}_{i v}^{2}, \\
Q & \equiv \sum_{i} \frac{I_{i}}{2 N} \dot{\bar{\theta}}_{i}^{2},
\end{aligned}
$$

where $v=x, y$ and $\dot{\overline{\mathbf{u}}}_{q} \equiv \overline{\mathbf{u}}_{q} / t_{0}$. The translational energy $K \equiv\left(K_{x}+K_{y}\right) / 2$ and rotational one $Q$ represent the intensity of the acoustic ( $\mathrm{L}$ and TR) modes and that of the RT mode, respectively. As shown in Fig. 6(a), if the stiffness ratio is large enough, $K$ and $Q$ dominate low $\left(\omega t_{0} \lesssim 1\right)$ and high $\left(\omega t_{0} \gtrsim 3\right)$ eigenfrequencies, respectively. In this case, the acoustic branches at low frequencies are well separated from the optical-like RT branch in the high frequency band (Fig. 3) where the shoulder of the vDOS [the dashed line in Fig. 6(a)] for high frequencies $3<\omega t_{0}<4.6$ is mostly owned 
(a)
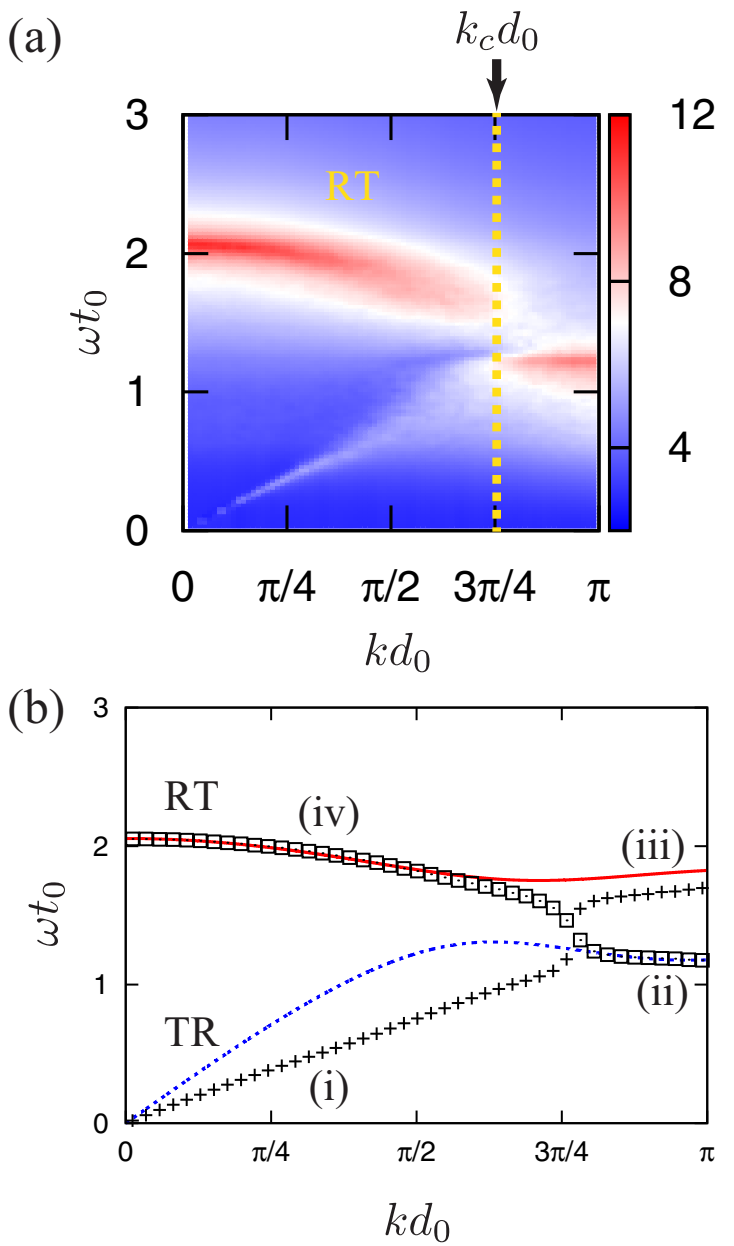

FIG. 5. (a) A three-dimensional (3D) plot of $\ln S_{r}(\omega, k)$ where the characteristic wave number $k_{c}$ is indicated by the arrow. (b) Dispersion relations obtained from Eq. (5) where the crosses and squares are $\omega_{t}(k)$ and $\omega_{r}(k)$, respectively. The dotted and solid lines represent the models Eqs. (6) and (7), respectively. In both (a) and (b), the stiffness ratio is $\rho=0.2$.

by micropolar rotations $Q$. However, if the stiffness ratio is small [Fig. 6(b)], there are four regions, i.e., (i) $K>Q$, (ii) $K<Q$, (iii) $K>Q$, and (iv) $K<Q$, corresponding to branches (i)-(iv) in Fig. 5(b). Thus, the transition from the optical-like to acoustic behavior of micropolar rotations is also evidenced by the eigenvectors (see also supporting data in Appendix G). In addition, the secondary peak of vDOS [the vertical arrow in Fig. 6(b)] represents the upper end of (ii), i.e., the RT mode on the acoustic branch.

\section{E. Frequency band of the RT mode}

The lattice-based model Eq. (7) also predicts frequency bands of the RT mode as

$$
\begin{aligned}
\omega_{r}\left(k_{\max }\right) & \leqslant \omega \leqslant \omega_{r}(0) \quad(\rho>1 / 7), \\
\omega_{r}(0) & \leqslant \omega \leqslant \omega_{r}\left(k_{\max }\right) \quad(\rho<1 / 7),
\end{aligned}
$$

where the limits coincide $\omega_{r}(0)=\omega_{r}\left(k_{\max }\right)$ at $\rho=1 / 7$ [6]. The shaded region in Fig. 7 is the model prediction of the frequency bands where the solid, dotted, and dashed lines
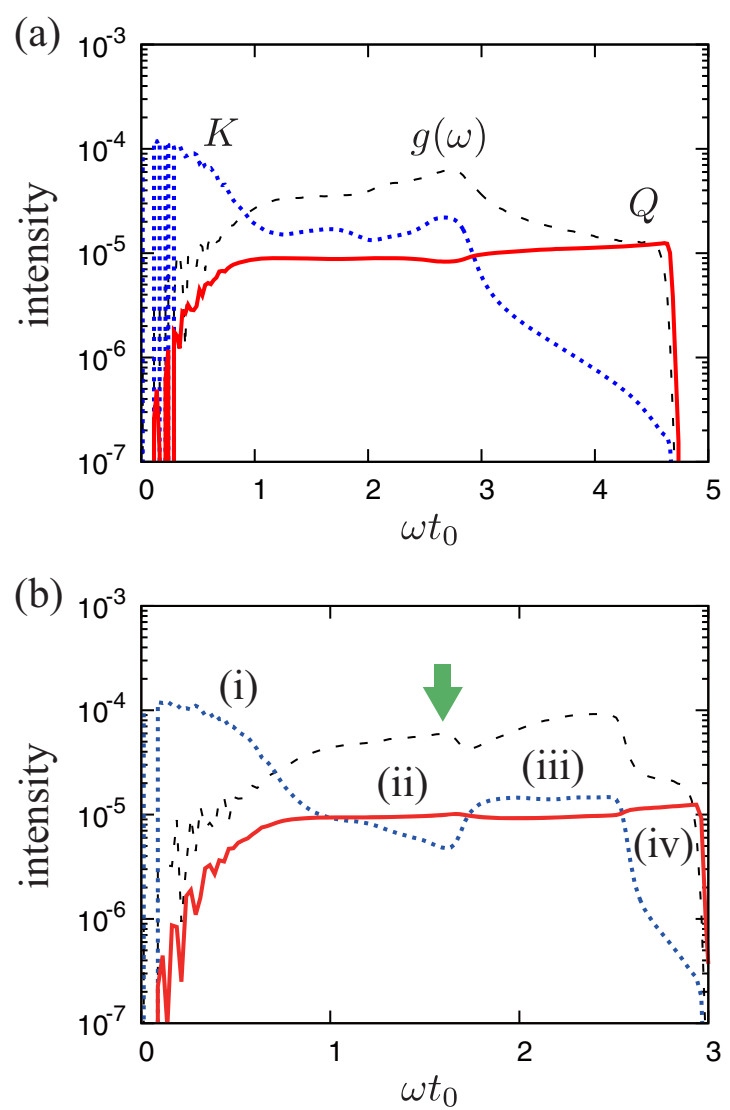

FIG. 6. Semilogarithmic plots of intensities of $K$ (blue dotted lines), $Q$ (red solid lines), and $g(\omega)$ multiplied by $5 \times 10^{-3}$ (dashed lines) where the stiffness ratios are given by (a) $\rho=1$ and (b) $\rho=0.4$. The green vertical arrow in (b) indicates the secondary peak of vDOS (as in Fig. 1).

represent

$$
\begin{aligned}
\omega_{r}(0) & =t_{0}^{-1} c_{r} \sqrt{40 \rho}, \\
\omega_{r}\left(k_{\max }\right) & =2 t_{0}^{-1} c_{r} \sqrt{3 \rho+1}, \\
\omega_{t}\left(k_{\max }\right) & =t_{0}^{-1} c_{t} \sqrt{10 \rho},
\end{aligned}
$$

respectively. Our numerical results of the limit $\omega_{r}(0)$ (circles) agree well with the model over the whole range of stiffness ratios. In addition, the cutoff frequency of vDOS $\omega_{c}$ (crosses) shows qualitatively the same behavior as $\omega_{r}(0)$, where $\omega_{c}$ is slightly higher because of the nonsharp upper limit of the dispersion relation. If the stiffness ratio is large $\rho>1$ numerical results of the limit $\omega_{r}\left(k_{\max }\right)$ (squares) are explained by the model. However, if the stiffness ratio is small $\rho<$ 1 , the limit switches to the acoustic branch, resulting from the transition of the RT mode (Fig. 5). Therefore, different from granular crystals [6], the upper and lower limits of the RT mode $\omega_{r}(0)$ and $\omega_{r}\left(k_{\max }\right)$ do not coincide. We also note that the limit frequency of the TR mode $\omega_{t}\left(k_{\max }\right)$ (triangles) slightly accedes to the model predictions.

\section{DISCUSSION}

In this paper, we have numerically investigated sound in two-dimensional disk systems with the focus on: (i) the 


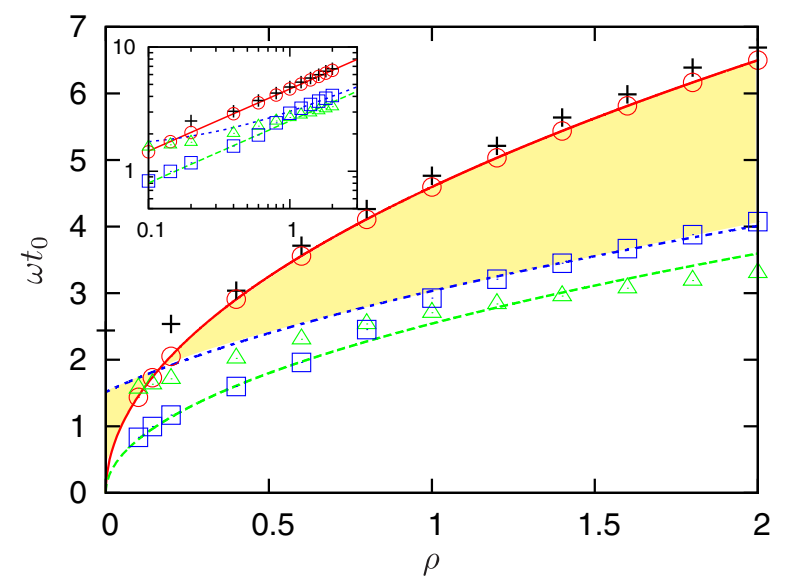

FIG. 7. Frequency bands of the RT mode where the red solid, blue dotted, and green dashed lines are the model predictions of $\omega_{r}(0), \omega_{r}\left(k_{\max }\right)$, and $\omega_{t}\left(k_{\max }\right)$, respectively [Eqs. (13)-(15)]. The shaded region is the model prediction of the frequency bands Eq. (12). The circles, squares, and triangles are numerical results of limit frequencies $\omega_{r}(0), \omega_{r}\left(k_{\max }\right)$, and $\omega_{t}\left(k_{\max }\right)$, respectively, whereas the crosses are the cutoff frequencies $\omega_{c}$ estimated from vDOS. The inset shows a double logarithmic plot where the red solid and green dashed lines have the slope $1 / 2$.

configurational disorder and (ii) the strength of tangential stiffness. A high frequency band or shoulder in the vDOS, which develops for higher frequencies with increasing the stiffness ratio (or tangential forces), is characteristic of disk systems involving rotational degrees of freedom. For strong tangential coupling, we observe that the RT mode exhibits much faster oscillations than those of the acoustic L and TR modes with a band gap in between. The fast oscillations of the RT mode (or micropolar rotations) are represented by an optical-like branch in the dispersion relation, which corresponds to the high frequency band in the vDOS. We explain the characteristic optical-like dispersion relations by introducing a modified lattice-based model, which perfectly describes our numerical results in the case that tangential forces are strong enough and in any case of small wave numbers. It is remarkable, however, that the RT mode exhibits a transition to the acoustic branch in the short wavelength regime if the tangential forces are comparable with or smaller than the normal forces. This transition is also evidenced by our analysis of eigenvectors and is related to the secondary peak in the vDOS.

Furthermore, even though an ideal Hertz-Mindlin contact would feature a fixed ratio $\rho=k_{t} / k_{n}$ between tangential and normal stiffnesses, we have in mind realistic contacts with imperfect possibly rough or inhomogeneous surfaces. Therefore, we cover a wide range of stiffness ratios instead of only one value also given the fact that we are in a 2D model system anyway where $\rho$ is different than in 3D. We also emphasize that some results, such as the transition between translational and rotational modes occurs for relatively small ratios $\rho$.

In our numerical simulations, we model both the normal and the tangential forces by elastic springs. Thus, the standing waves presented are purely elastic so that total energy is conserved throughout simulations. This means that the decay of the VAFs is solely caused by scattering attenuation (and not by energy dissipation). However, in reality, dissipative forces, e.g., the Coulomb or sliding friction and viscous forces, also exist between the disks in contact. To take into account such dissipation of energy requires some generalizations of our model $[40,41]$ as left to future work. Similarly, it is interesting to study how other interaction forces, e.g., the rolling resistance [6] and the attractive interaction due to capillary bridges in wet granular material [42-44], affect the results. Moreover, the influence of microstructure [1], e.g., size distributions and polydispersity, on the rotational sound requires more research. For practical purposes, numerical studies in three dimensions are crucial where an additional degree of freedom, i.e., the twisting motion of spheres in contact, enables a pure rotational $(\mathrm{R})$ mode $[6,9]$. Therefore, further studies are needed to clarify how configurational disorder affects the L, TR, RT, and $\mathrm{R}$ modes in three-dimensional granular media. In addition, wave diffusion [45] and localization phenomena [46,47] are also important aspects of sound in granular material.

\section{CONCLUSION}

We conclude that rotational sound exhibits a characteristic optical-like dispersion relation even in disordered systems. If the tangential forces are weak, it flips to the acoustic branch at a characteristic wavelength so that configurational disorder enables the acousticlike behavior of micropolar rotations on small enough scales. Although rotational waves are usually not propagating well in most systems, this transition from rotational to acoustic modes of wave propagation might be useful for designing (meta) materials that do feature rotational wave transport over long distances.

\section{ACKNOWLEDGMENTS}

We thank A. Merkel, X. Jia, H. Mizuno, B. P. Tighe, V. Magnanimo, and $\mathrm{H}$. Cheng for fruitful discussions. This work was financially supported by the Kawai Foundation for Sound Technology \& Music and KAKENHI Grants No. 16H04025 and No. 18K13464 from JSPS. A part of the computation has been carried out at the Yukawa Institute Computer Facility.

\section{APPENDIX A: MASS MATRIX AND DYNAMICAL MATRIX}

In this appendix, we show the details of the mass matrix and dynamical matrix in Eq. (1).

The $3 N \times 3 N$ mass matrix is given by

$$
\mathcal{M}=\left(\begin{array}{ccc}
m_{i} & 0 & 0 \\
0 & m_{i} & 0 \\
0 & 0 & I_{i}
\end{array}\right)_{i=1, \ldots, N}
$$

where $m_{i}$ and $I_{i}=m_{i} d_{i}^{2} / 8$ with the disk diameter $d_{i}$ are the mass and moment of inertia of the disk $i$, respectively. 
The $3 N \times 3 N$ Hessian is given by second derivatives of the elastic energy $E$ as

$$
\mathcal{D}=\left(\begin{array}{lll}
\frac{\partial^{2} E}{\partial x_{i} \partial x_{j}} & \frac{\partial^{2} E}{\partial x_{i} \partial y_{j}} & \frac{\partial^{2} E}{\partial x_{i} \partial \theta_{j}} \\
\frac{\partial^{2} E}{\partial y_{i} \partial x_{j}} & \frac{\partial^{2} E}{\partial y_{i} \partial y_{j}} & \frac{\partial^{2} E}{\partial y_{i} \partial \theta_{j}} \\
\frac{\partial^{2} E}{\partial \theta_{i} \partial x_{j}} & \frac{\partial^{2} E}{\partial \theta_{i} \partial y_{j}} & \frac{\partial^{2} E}{\partial \theta_{i} \partial \theta_{j}}
\end{array}\right)_{i, j=1, \ldots, N} .
$$

We introduce elastic energy as the sum of pairwise potentials, i.e., $E=\sum_{i>j} e_{i j}$. The pairwise potential is decomposed into harmonic potentials stored in the normal and tangential directions as

$$
e_{i j}=\frac{k_{n}}{2} \xi_{i j}^{2}+\frac{k_{t}}{2} \mathbf{u}_{i j}^{\perp 2},
$$

where $k_{n}$ and $k_{t}$ are the normal and tangential stiffnesses, respectively. In Eq. (A3), $\xi_{i j} \equiv\left(d_{i}+d_{j}\right) / 2-r_{i j}>0$ represents the overlap between the disks $(i$ and $j$ ) in contact, where $r_{i j} \equiv\left|\mathbf{r}_{i j}\right|$ with the relative position between the disks $\mathbf{r}_{i j} \equiv \mathbf{r}_{i}-\mathbf{r}_{j}$ is the interparticle distance. In addition,

$$
\mathbf{u}_{i j}^{\perp} \equiv \mathbf{u}_{i j}-\mathbf{u}_{i j}^{\|}-\boldsymbol{\theta}_{i j} \times \mathbf{n}_{i j}
$$

is the relative displacement in the tangential direction where we introduced relative displacements as

$$
\begin{aligned}
\mathbf{u}_{i j} & \equiv \mathbf{u}_{i}-\mathbf{u}_{j}, \\
\mathbf{u}_{i j}^{\|} & \equiv\left(\mathbf{u}_{i j} \cdot \mathbf{n}_{i j}\right) \mathbf{n}_{i j}, \\
\boldsymbol{\theta}_{i j} & \equiv \frac{1}{2}\left(d_{i} \theta_{i}+d_{j} \theta_{j}\right) \mathbf{n}_{z},
\end{aligned}
$$

with the normal unit vector $\mathbf{n}_{i j} \equiv \mathbf{r}_{i j} / r_{i j}$ and out of the $x y$ plane unit vector $\mathbf{n}_{z}$ (parallel to the $z$ axis). Then, the second derivatives of Eq. (A3) are given by

$$
\begin{aligned}
& \frac{\partial^{2} e_{i j}}{\partial x_{i} \partial x_{i}}= k_{n}-k_{n}\left(1+\frac{\xi_{i j}}{r_{i j}}-\rho\right) n_{i j y}^{2}, \\
& \frac{\partial^{2} e_{i j}}{\partial x_{i} \partial y_{i}}=k_{n}\left(1+\frac{\xi_{i j}}{r_{i j}}-\rho\right) n_{i j x} n_{i j y}, \\
& \frac{\partial^{2} e_{i j}}{\partial y_{i} \partial y_{i}}=k_{n}-k_{n}\left(1+\frac{\xi_{i j}}{r_{i j}}-\rho\right) n_{i j x}^{2}, \\
& \frac{\partial^{2} e_{i j}}{\partial x_{i} \partial \theta_{i}}=\frac{k_{t}}{2} d_{i} n_{i j y}, \\
& \frac{\partial^{2} e_{i j}}{\partial y_{i} \partial \theta_{i}}=-\frac{k_{t}}{2} d_{i} n_{i j x}, \\
& \frac{\partial^{2} e_{i j}}{\partial \theta_{i} \partial \theta_{i}}=\frac{k_{t}}{4} d_{i}^{2},
\end{aligned}
$$

where the $x$ and $y$ components are written as $\mathbf{r}_{i}=\left(x_{i}, y_{i}\right)$, and $\mathbf{n}_{i j}=\left(n_{i j x}, n_{i j y}\right)$. Note that the second derivatives with different indices ( $i$ and $j$ ) are related to Eqs. (A8)-(A13) as

$$
\begin{aligned}
\frac{\partial^{2} e_{i j}}{\partial \alpha_{i} \partial \beta_{j}} & =-\frac{\partial^{2} e_{i j}}{\partial \alpha_{i} \partial \beta_{i}}, \\
\frac{\partial^{2} e_{i j}}{\partial \alpha_{i} \partial \theta_{j}} & =\frac{d_{j}}{d_{i}} \frac{\partial^{2} e_{i j}}{\partial \alpha_{i} \partial \theta_{i}}, \\
\frac{\partial^{2} e_{i j}}{\partial \theta_{i} \partial \theta_{j}} & =\frac{d_{j}}{d_{i}} \frac{\partial^{2} e_{i j}}{\partial \theta_{i} \partial \theta_{i}},
\end{aligned}
$$

where $\alpha, \beta=x, y$.

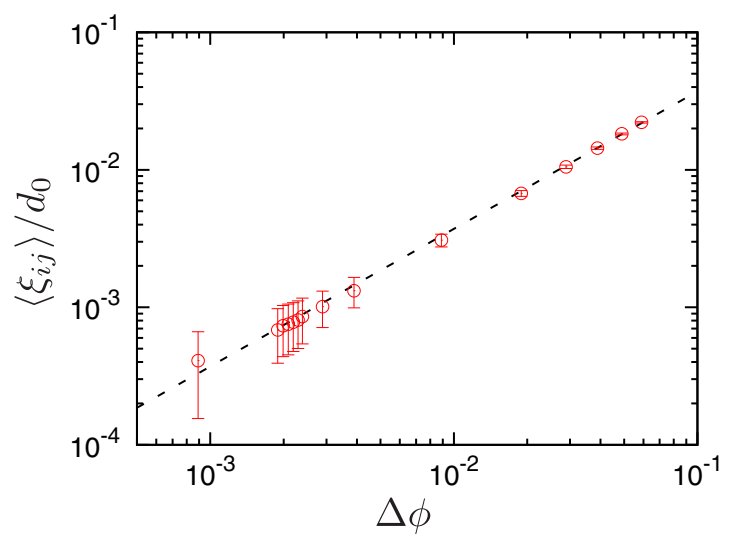

FIG. 8. A double logarithmic plot of the average overlap scaled by the mean disk diameter $\left\langle\xi_{i j}\right\rangle / d_{0}$ (circles) where the horizontal axis is the proximity to jamming $\Delta \phi$. The dotted line represents the linear relation $\left\langle\xi_{i j}\right\rangle / d_{0}=A \Delta \phi$ where the proportionality and the jamming transition density are given by $A \simeq 0.3715$ and $\phi_{J} \simeq$ 0.8411 , respectively. Here, we take sample averages of $\left\langle\xi_{i j}\right\rangle$ over $10^{3}$ different configurations of $N=2048$ frictionless disks.

\section{APPENDIX B: DISORDERED CONFIGURATIONS}

In this appendix, we explain how to prepare initial disordered configurations by MD simulations.

To avoid crystallization, we randomly distribute a 50:50 binary mixture of $N$ frictionless disks $\left(k_{t}=0\right)$ in a periodic box where different kinds of disks have the same mass $m$ and different diameters $d_{L}$ and $d_{S}$ (with ratio $d_{L} / d_{S}=1.4$ ). Then, we minimize elastic energy $E_{n}=\sum_{i>j} k_{n} \xi_{i j}^{2} / 2$ with the aid of the FIRE algorithm [48] and stop the energy minimization once the maximum of disk accelerations becomes less than $10^{-6} k_{n} d_{0} / \mathrm{m}$. During the energy minimization, equations of motion are numerically integrated by the velocity Verlet scheme where the time increment can increase from the initial value $\Delta t_{\text {ini }}=10^{-2} \sqrt{m / k_{n}}$ to the maximum $\Delta t_{\max }=10 \Delta t_{\text {ini }}$, according to the FIRE algorithm [48]. In addition, we use recommended values for other parameters $\left(N_{\min }=5, f_{\text {inc }}=\right.$ 1.1, $f_{\text {dec }}=0.5, \alpha_{\text {start }}=0.1$, and $f_{\alpha}=0.99$ as in Ref. [48]) in FIRE. After the energy minimization, no potential energy is stored in tangential direction so that the system is still in mechanical equilibrium even if we switch on the tangential forces, i.e., $E=E_{n}$, even though $k_{t}>0$. Therefore, our systems are initially unstressed in the tangential direction [49]. Note that stressed systems can be made if we prepare initial disordered configurations with tangential forces $\left(k_{t}>\right.$ 0 ) which make the configurations history dependent. Figure 8 displays a double logarithmic plot of the average overlap between the disks in contact $\left\langle\xi_{i j}\right\rangle$. We confirm, in our disordered (frictionless) disk packings, the well-known critical scaling $\left\langle\xi_{i j}\right\rangle \sim \Delta \phi$, where $\Delta \phi \equiv \phi-\phi_{J}$ is the proximity to the jamming transition density $\phi_{J}$.

\section{APPENDIX C: THE VAFS AND POWER SPECTRA}

In this appendix, we summarize the details of the VAFs and power spectra. 
We calculate Fourier transforms of disks' velocities as

$$
\left\{\dot{\mathbf{u}}_{\mathbf{k}}(t), \dot{\theta}_{\mathbf{k}}(t)\right\}=\sum_{i=1}^{N}\left\{\dot{\mathbf{u}}_{i}(t), \dot{\theta}_{i}(t)\right\} e^{-I \mathbf{k} \cdot \mathbf{r}_{i}(t)},
$$

where the position $\mathbf{r}_{i}(t)$ is also given by numerical solutions of Eq. (1). The L and TR velocities are defined as

$$
\begin{gathered}
\dot{\mathbf{u}}_{\mathbf{k}}^{\|}(t) \equiv\left\{\dot{\mathbf{u}}_{\mathbf{k}}(t) \cdot \hat{\mathbf{k}}\right\} \hat{\mathbf{k}}, \\
\dot{\mathbf{u}}_{\mathbf{k}}^{\perp}(t) \equiv \dot{\mathbf{u}}_{\mathbf{k}}(t)-\dot{\mathbf{u}}_{\mathbf{k}}^{\|}(t),
\end{gathered}
$$

respectively, where $\hat{\mathbf{k}} \equiv \mathbf{k} / k$ is a unit vector. Then, the normalized VAFs of L, TR, and RT velocities are given by

$$
\begin{gathered}
C_{l}(k, t)=\frac{\left\langle\dot{\mathbf{u}}_{\mathbf{k}}^{\|}(t) \cdot \dot{\mathbf{u}}_{-\mathbf{k}}^{\|}(0)\right\rangle}{\left\langle\left|\dot{\mathbf{u}}_{\mathbf{k}}^{\|}(0)\right|^{2}\right\rangle}, \\
C_{t}(k, t)=\frac{\left\langle\dot{\mathbf{u}}_{\mathbf{k}}^{\perp}(t) \cdot \dot{\mathbf{u}}_{-\mathbf{k}}^{\perp}(0)\right\rangle}{\left\langle\left|\dot{\mathbf{u}}_{\mathbf{k}}^{\perp}(0)\right|^{2}\right\rangle}, \\
C_{r}(k, t)=\frac{\left\langle\dot{\theta}_{\mathbf{k}}(t) \dot{\theta}_{-\mathbf{k}}(0)\right\rangle}{\left\langle\left|\dot{\theta}_{\mathbf{k}}(0)\right|^{2}\right\rangle},
\end{gathered}
$$

respectively.

The power spectra of L, TR, and RT velocities are introduced as

$$
\begin{aligned}
& S_{l}(k, \omega)=\left\langle\left|\tilde{\mathbf{u}}_{\mathbf{k}}^{\|}(\omega)\right|^{2}\right\rangle, \\
& S_{t}(k, \omega)=\left\langle\left|\tilde{\mathbf{u}}_{\mathbf{k}}^{\perp}(\omega)\right|^{2}\right\rangle, \\
& S_{r}(k, \omega)=\left\langle\left|\tilde{\theta}_{\mathbf{k}}(\omega)\right|^{2}\right\rangle,
\end{aligned}
$$

respectively, where the Fourier transforms are given by

$$
\begin{aligned}
\tilde{\mathbf{u}}_{\mathbf{k}}^{\|}(\omega) & \equiv \int_{0}^{\infty} \dot{\mathbf{u}}_{\mathbf{k}}^{\|}(t) e^{I \omega t} d t, \\
\tilde{\mathbf{u}}_{\mathbf{k}}^{\perp}(\omega) & \equiv \int_{0}^{\infty} \dot{\mathbf{u}}_{\mathbf{k}}^{\perp}(t) e^{I \omega t} d t, \\
\tilde{\theta}_{\mathbf{k}}(\omega) & \equiv \int_{0}^{\infty} \dot{\theta}_{\mathbf{k}}(t) e^{I \omega t} d t .
\end{aligned}
$$

\section{APPENDIX D: DEPENDENCE OF VIBRATIONAL DENSITY OF STATES ON THE AREA FRACTION}

In this appendix, we show the dependence of vDOS on the area fraction $\phi$.

Figure 9 displays the vDOS, where the stiffness ratio is fixed to $\rho=1$ and the area fraction increases from $\phi=$ 0.8435 to 0.9 . The distance from jamming for frictionless disks is limited to $\phi-\phi_{J}>10^{-3}$, and the vDOS is quite insensitive to the area fraction.

\section{APPENDIX E: PHASE SPEEDS AND ATTENUATION COEFFICIENTS OF ACOUSTIC MODES}

In this appendix, we present phase speeds $c_{\alpha}(k) \equiv$ $\omega_{\alpha}(k) / k$ and attenuation coefficients $\gamma_{\alpha}(k)$ obtained from the fitting of damped oscillations Eq. (5) to the VAFs of the acoustic $\mathrm{L}$ and TR modes $(\alpha=l, t)$.

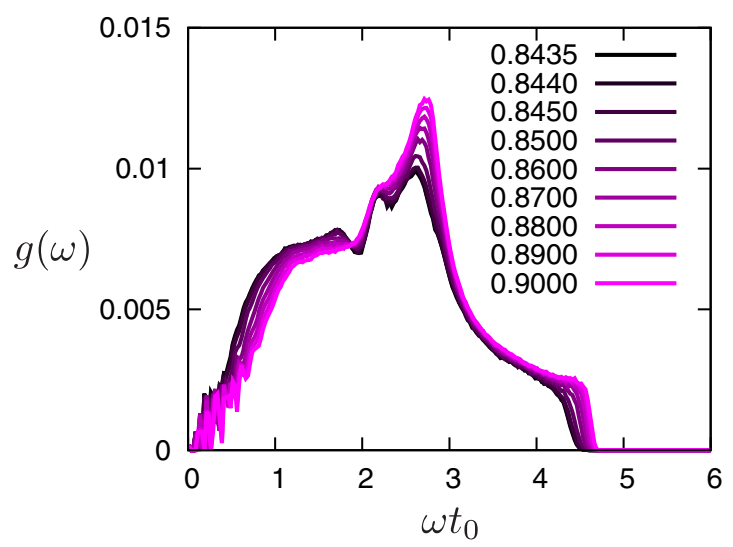

FIG. 9. The vDOS of disordered disk systems with tangential elastic forces (solid lines) where the stiffness ratio is $\rho=1$ and the area fraction increases as listed in the legend.

Figure 10 displays the phase speeds of (a) $\mathrm{L}$ and (b) TR modes where the area fraction is fixed at $\phi=0.9$ and the stiffness ratio $\rho$ increases as indicated by the arrows. The phase speeds shift to higher values with increasing $\rho$ and small dips can be found around $k d_{0} \simeq 1$ as reported in Refs. [16,17]. As shown in Fig. 11, the attenuation power law is well explained by the Rayleigh prediction in twodimensional $\gamma_{\alpha}(k) \sim k^{3}$ (dashed lines). However, if $\rho=0$ (if
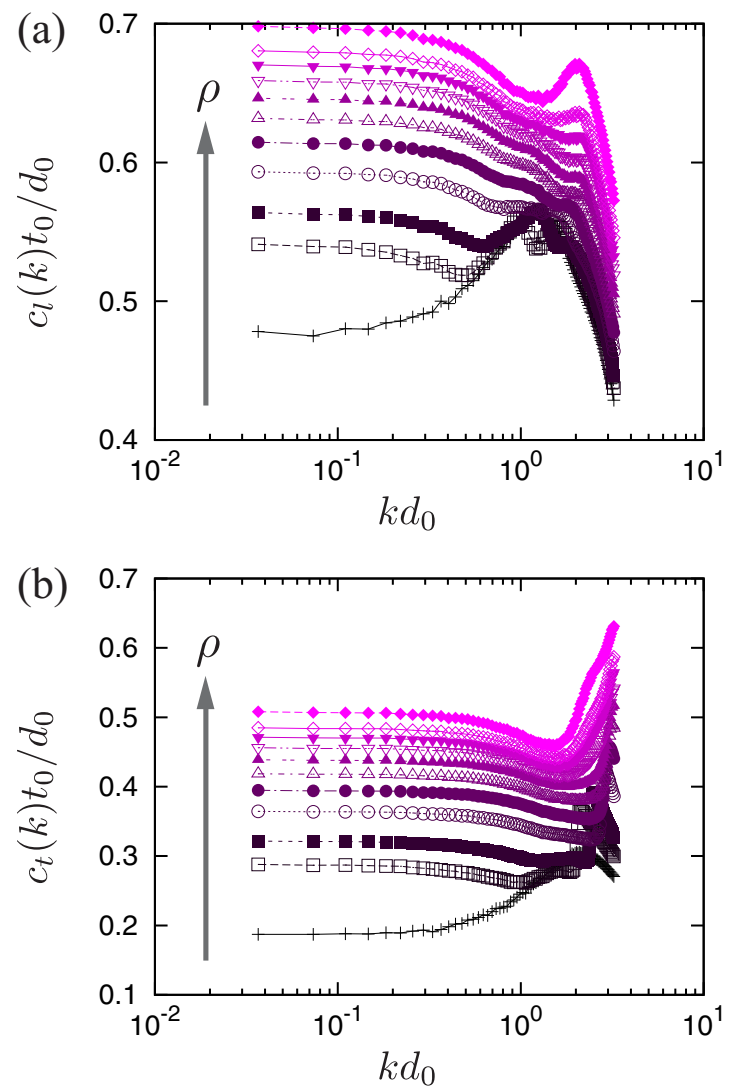

FIG. 10. Semilogarithmic plots of the phase speeds of (a) L and (b) TR modes where the area fraction is $\phi=0.9$. The stiffness ratio increases as $\rho=0,0.1,0.2,0.4,0.6,0.8,1.0,1.2,1.4,1.6$, and 2.0 (as indicated by the arrows). 

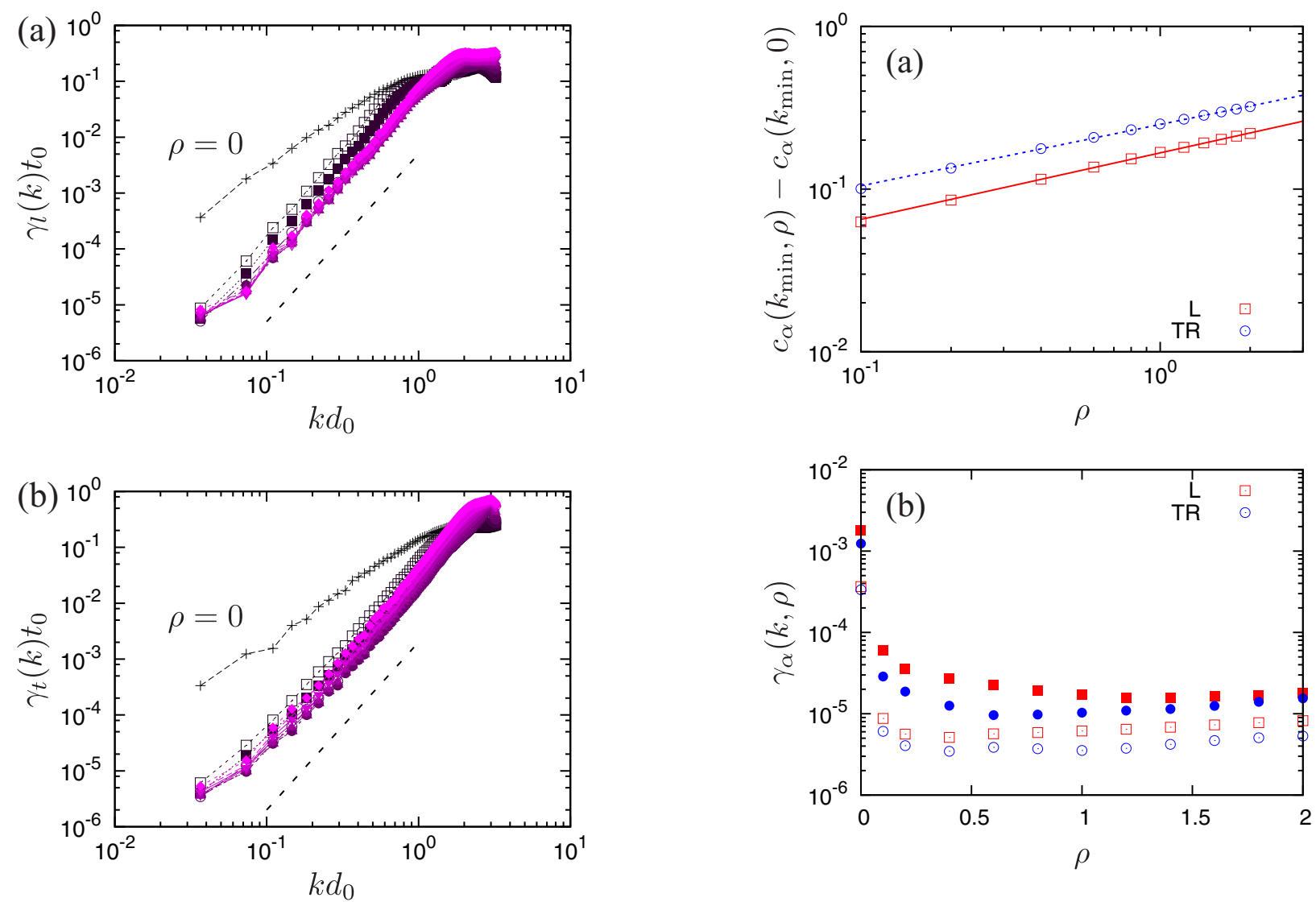

FIG. 11. Double logarithmic plots of the attenuation coefficients of (a) the L and (b) the TR modes where the area fraction and stiffness ratio are as in Fig. 10. The dashed lines represent the Rayleigh prediction in two-dimensional $\gamma_{\alpha}(k) \sim k^{3}(\alpha=l, t)$.

the disks are frictionless), we observe a considerable deviation from the Rayleigh prediction as reported in Ref. [16].

The phase speeds and attenuation coefficients depend on the stiffness ratio as $c_{\alpha}(k, \rho)$ and $\gamma_{\alpha}(k, \rho)$. Here, we report their dependence on the stiffness ratio by taking their
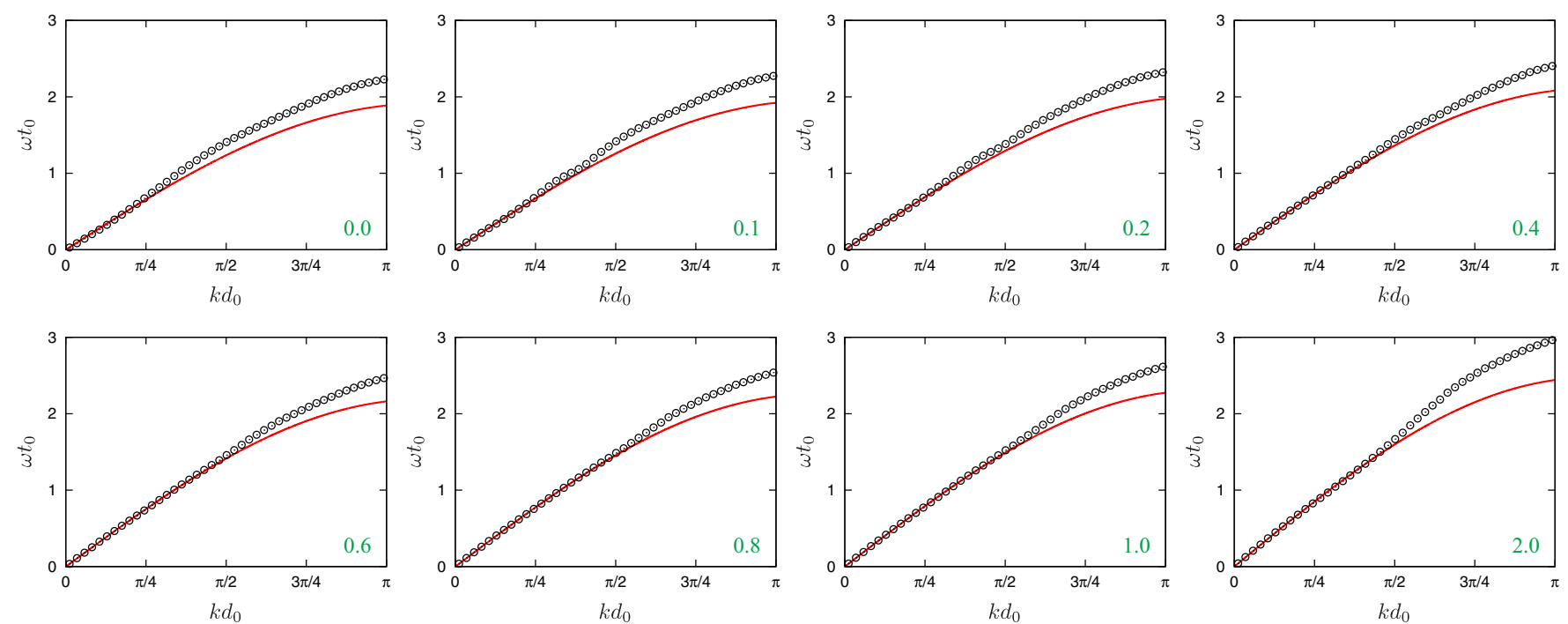

FIG. 13. Dispersion relations of the L mode (circles) where the lines are model predictions in long wavelengths [Eq. (F1) fitted to the range between $\left.0<k d_{0}<\pi / 4\right]$. The area fraction is fixed at $\phi=0.9$, whereas the stiffness ratio changes as displayed in each panel (right bottom). 

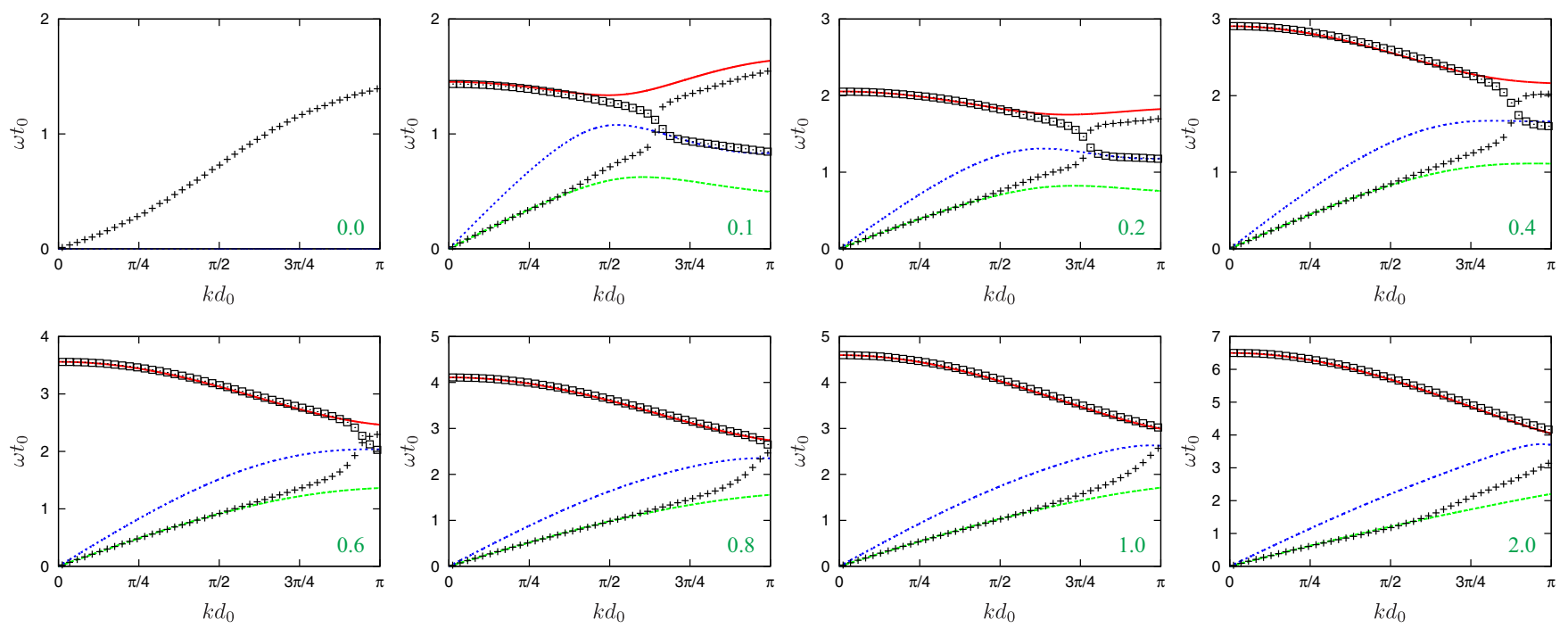

FIG. 14. Dispersion relations of the TR (pluses) and RT (squares) modes where the area fraction and stiffness ratio are as in Fig. 13. The blue dotted and red solid lines are the model predictions Eqs. (6) and (7), respectively, whereas the green dashed lines is Eq. (6) fitted to the data of the TR mode in long wavelengths $\left(0<k d_{0}<\pi / 4\right)$.

increase with increasing the stiffness ratio where the lines $c_{\alpha}\left(k_{\min }, \rho\right)-c_{\alpha}\left(k_{\min }, 0\right) \sim \rho^{0.4}$ are guides to the eyes [note that the $\mathrm{L}$ mode is always faster than the TR mode, $c_{l}\left(k_{\min }, \rho\right)>c_{t}\left(k_{\min }, \rho\right)$ for $\left.0 \leqslant \rho \leqslant 2\right]$. The open symbols in Fig. 12(b) are the attenuation coefficients at the smallest wave number $\gamma_{\alpha}\left(k_{\min }, \rho\right)$, whereas the closed symbols are the results at the second smallest wave number $\gamma_{\alpha}\left(2 k_{\min }, \rho\right)$.

\section{APPENDIX F: LATTICE-BASED MODEL AND DISPERSION RELATIONS}

In this appendix, we explain the modified lattice model of the $\mathrm{L}$ mode and show additional data of dispersion relations.

In Ref. [6], Merkel and Luding studied vibrations of particles on a fcc lattice. The fcc structure can be seen as a dense stack of square layers ( $A$ and $B$ in the sequence $A B A B \cdots$ ), and they are considered sound propagation along the axis perpendicular to the layers. Assuming that every particle has the same mass and diameter, they calculated normal and tangential forces between the particles in contact. The contact forces were modeled by linear elastic springs, and dispersion relations were derived for the L, TR, and RT modes. We modify their results to describe dispersion relations in disordered disk systems where the acoustic branch for the $\mathrm{L}$ mode is given by

$$
\omega_{l}(k)=a_{l} \sqrt{8(\rho+1)} \sin (k l)
$$

In Eqs. (6), (7), and (F1), the prefactor $a_{\alpha}(\alpha=l, t, r)$ is introduced to represent the decrease in sound speed in disordered systems ( $a_{\alpha} \equiv t_{0}^{-1}$ in the case of the fcc structure [6]).

Figure 13 displays the dispersion relations of the $\mathrm{L}$ mode where we change the stiffness ratio from $\rho=0$ to 2 as shown in each panel (right bottom). The circles are obtained by fitting damped oscillations Eq. (5) to the VAFs of the L mode [Fig. 2(a)]. The lines are model predictions in long wavelengths where we fit Eq. (F1) to the numerical results of $\omega_{l}(k)$ in the range between $0<k d_{0}<\pi / 4$. Note that the prefactor $a_{l}$ in Eq. (F1) is adjusted to the data whereas the length scale $l=0.446 d_{0}$ is independent of the stiffness ratio. As shown in Fig. 15, $a_{l}$ (squares) monotonously decreases, and the factor $a_{l} / \sqrt{\rho+1}$ (triangles) further decreases with increasing the stiffness ratio.

Figure 14 shows the dispersion relations of the TR (pluses) and RT modes (squares) obtained by fitting Eq. (5) to the VAFs. The green dashed lines represent the model predictions of the TR mode in long wavelengths [Eq. (6) fitted to the data in $\left.0<k d_{0}<\pi / 4\right]$. The length scale $l=0.446 d_{0}$ is used for the whole stiffness ratios, whereas the prefactor $a_{t}$ is adjusted to the data (see the circles in Fig. 15). The dispersion relations of the RT mode are well described by the model Eq. (7) (red solid lines) if the stiffness ratio is large $(\rho \geqslant 0.8)$ or the wave number is small enough. Importantly, both the length scale $\left(l=0.446 d_{0}\right)$ and the prefactor $\left(a_{r}=0.73 t_{0}^{-1}\right)$ are independent of the stiffness ratio. If the stiffness ratio is small $(\rho<0.8)$, the RT mode exhibits a transition from the optical-like to the acoustic branches. Then, the RT mode in the acoustic branch is well captured by the model prediction of

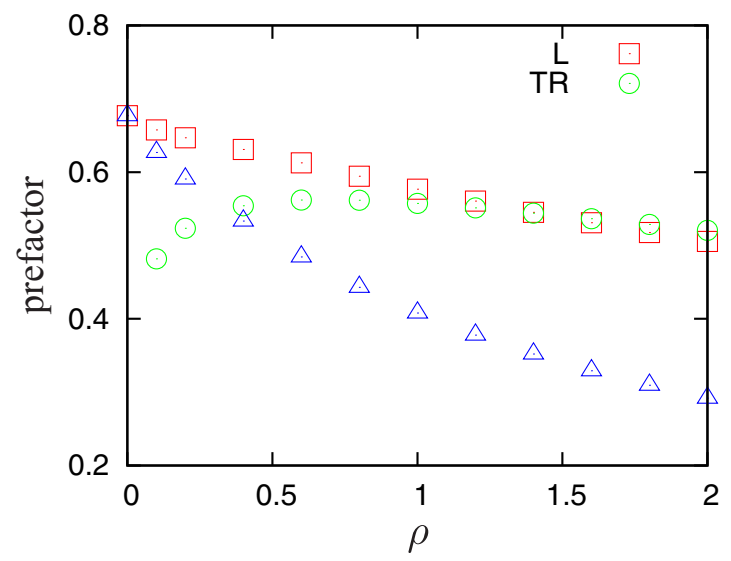

FIG. 15. Prefactors, $a_{l}$ (squares) and $a_{t}$ (circles) in Eqs. (F1) and (6) adjusted to the data in long wavelengths $\left(0<k d_{0}<\pi / 4\right)$. The triangles are the factor $a_{l} / \sqrt{\rho+1}$. 

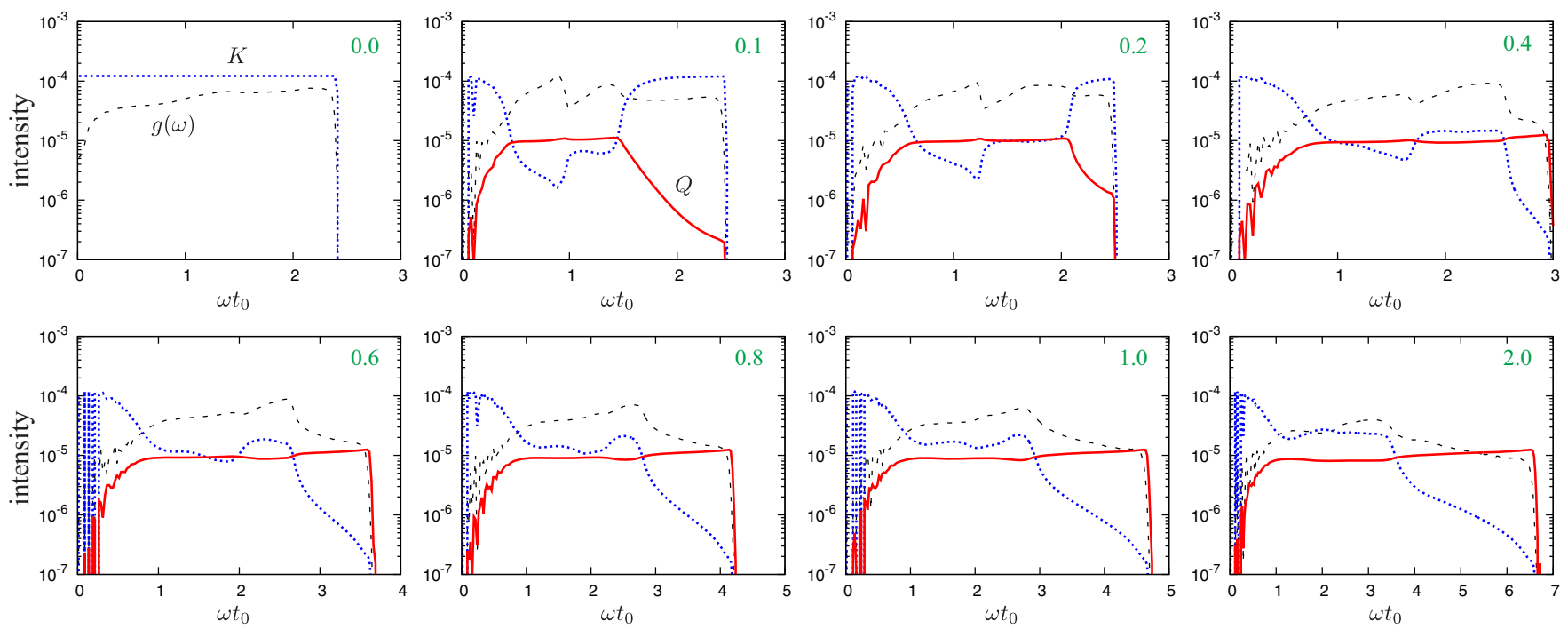

FIG. 16. Semilogarithmic plots of intensities of the translational energy $K$ (blue dotted lines) and rotational one $Q$ (red solid lines) where the dashed lines are the vDOS $g(\omega)$ multiplied by $5 \times 10^{-3}$. The area fraction and stiffness ratio are as in Fig. 13 .

the TR mode Eq. (6) (blue dotted lines) where the length scale and prefactor are modified as $l=0.516 d_{0}$ and $a_{t}=0.83 t_{0}^{-1}$ independently of the stiffness ratio. In frictionless systems, the RT mode does not exist, and the model prediction of the TR mode is always zero ( $\rho=0$ in Fig. 14).

\section{APPENDIX G: TRANSLATIONAL AND ROTATIONAL ENERGIES}

In this appendix, we present supporting data of the eigenvector analyses.
Figure 16 shows the intensities of $K$ (blue dotted lines) and $Q$ (red solid lines) where we also plot the vDOS $g(\omega)$ multiplied by $5 \times 10^{-3}$ (dashed lines). The stiffness ratio $\rho$ changes as displayed in each panel (right top), where $K(Q)$ dominates lower (higher) frequencies if the stiffness ratio is large enough $\rho \geqslant 0.8$. In the case of $\rho=0$ (frictionless disks), the micropolar rotations do not exist, i.e., $\bar{\theta}_{i}=0$, and the translational energy is constant $K \simeq 10^{-4}$ because the eigenvectors are normalized as $\left|\overline{\mathbf{u}}_{q}\right|^{2}=\sum_{i=1}^{N}\left(\bar{u}_{i x}^{2}+\bar{u}_{i y}^{2}\right)=$ const.
[1] P. Sheng, Introduction to Wave Scattering, Localization and Mesoscopic Phenomena (Springer-Verlag, Berlin/Heidelberg, 2006).

[2] H. Sato, M. C. Fehler, and T. Maeda, Seismic Wave Propagation and Scattering in the Heterogeneous Earth (Springer-Verlag, Berlin/Heidelberg, 2012).

[3] E. Cosserat and F. Cosserat, Théorie des Corps Déformables (Hermann et Fils, Paris, 1909).

[4] L. M. Schwartz, D. L. Johnson, and S. Feng, Phys. Rev. Lett. 52, 831 (1984).

[5] A. Merkel, V. Tournat, and V. Gusev, Phys. Rev. Lett. 107, 225502 (2011).

[6] A. Merkel and S. Luding, Int. J. Solids Struct. 106-107, 91 (2017).

[7] J. O'Donovan, E. Ibraim, C. O'Sullivan, S. Hamlin, D. M. Wood, and G. Marketos, Granular Matter 18, 56 (2016).

[8] J. O’Donovan, C. O’Sullivan, G. Marketos, and D. M. Wood, Granular Matter 17, 197 (2015).

[9] A. Merkel, V. Tournat, and V. Gusev, Phys. Rev. E 82, 031305 (2010).

[10] S. Chakravarty and S. Sen, Granular Matter 20, 42 (2018).

[11] R. K. Shrivastava and S. Luding, Nonlinear Process. Geophys. 24, 435 (2017).
[12] B. P. Lawney and S. Luding, Acta Mech. 225, 2385 (2014).

[13] O. Mouraille and S. Luding, Ultrasonics 48, 498 (2008).

[14] G. Baldi, V. M. Giordano, G. Monaco, and B. Ruta, Phys. Rev. Lett. 104, 195501 (2010).

[15] G. Baldi, V. M. Giordano, B. Ruta, R. Dal Maschio, A. Fontana, and G. Monaco, Phys. Rev. Lett. 112, 125502 (2014).

[16] S. Gelin, H. Tanaka, and A. Lemaître, Nat. Mater. 15, 1177 (2016).

[17] G. Monaco and S. Mossa, Proc. Natl. Acad. Sci. USA 106, 16907 (2009).

[18] H. Mizuno and R. Yamamoto, Phys. Rev. Lett. 110, 095901 (2013).

[19] A. Marruzzo, W. Schirmacher, A. Fratalocchi, and G. Ruocco, Sci. Rep. 3, 1407 (2013).

[20] G. Monaco and V. M. Giordano, Proc. Natl. Acad. Sci. USA 106, 3659 (2009).

[21] V. Lubchenko and P. G. Wolynes, Proc. Natl. Acad. Sci. USA 100, 1515 (2003).

[22] T. S. Grigera, V. Martin-Mayor, G. Parisi, and P. Verrocchio, Nature (London) 422, 289 (2003).

[23] W. Schirmacher, G. Ruocco, and T. Scopigno, Phys. Rev. Lett. 98, 025501 (2007).

[24] H. Mizuno, H. Shiba, and A. Ikeda, Proc. Natl. Acad. Sci. USA 114, E9767 (2017). 
[25] M. L. Manning and A. J. Liu, Phys. Rev. Lett. 107, 108302 (2011).

[26] M. L. Manning and A. J. Liu, Europhys. Lett. 109, 36002 (2015).

[27] L. E. Silbert, A. J. Liu, and S. R. Nagel, Phys. Rev. Lett. 95, 098301 (2005).

[28] M. Wyart, L. E. Silbert, S. R. Nagel, and T. A. Witten, Phys. Rev. E 72, 051306 (2005).

[29] M. Wyart, S. R. Nagel, and T. A. Witten, Europhys. Lett. 72, 486 (2005).

[30] L. E. Silbert, A. J. Liu, and S. R. Nagel, Phys. Rev. E 79, 021308 (2009).

[31] V. F. Nesterenko, J. Appl. Mech. Tech. Phys. 24, 733 (2012).

[32] L. R. Gómez, A. M. Turner, M. van Hecke, and V. Vitelli, Phys. Rev. Lett. 108, 058001 (2012).

[33] L. R. Gómez, A. M. Turner, and V. Vitelli, Phys. Rev. E 86, 041302 (2012).

[34] S. Ulrich, N. Upadhyaya, B. van Opheusden, and V. Vitelli, Proc. Natl. Acad. Sci. USA 110, 20929 (2013).

[35] A. M. Tichler, L. R. Gómez, N. Upadhyaya, X. Campman, V. F. Nesterenko, and V. Vitelli, Phys. Rev. Lett. 111, 048001 (2013).

[36] N. Upadhyaya, L. R. Gómez, and V. Vitelli, Phys. Rev. X 4, 011045 (2014).

[37] Due to the tangential force and interlocking of the disks, every mode is excited by any combinations of the amplitudes and wave vectors. For example, the combination in the first line of
Table I excites not only the L mode, but also the TR and RT modes, although their intensities are quite weak.

[38] K. Saitoh, V. Magnanimo, and S. Luding, Soft Matter 11, 1253 (2015).

[39] The Fourier transform of Eq. (5) is the Lorentzian $S_{\alpha}(k, \omega)=$ $\gamma_{\alpha}(k) /\left[\left\{\omega-\omega_{\alpha}(k)\right\}^{2}+\gamma_{\alpha}(k)^{2}\right]$, which also well describes the power spectra (Fig. 3) except for the weak optical-like and acoustic branches in Figs. 3(b) and 3(c), respectively.

[40] E. Somfai, M. van Hecke, W. G. Ellenbroek, K. Shundyak, and W. van Saarloos, Phys. Rev. E 75, 020301(R) (2007).

[41] D. L. Johnson, Y. Hu, and H. Makse, Phys. Rev. E 91, 062208 (2015).

[42] T. Brunet, X. Jia, and P. Mills, Phys. Rev. Lett. 101, 138001 (2008).

[43] A. Singh, V. Magnanimo, K. Saitoh, and S. Luding, Phys. Rev. E 90, 022202 (2014).

[44] R. Sudeshna, S. Luding, and T. Weinhart, New J. Phys. 19, 043014 (2017).

[45] X. Jia, Phys. Rev. Lett. 93, 154303 (2004).

[46] H. Hu, A. Strybulevych, J. H. Page, S. E. Skipetrov, and B. A. van Tiggelen, Nat. Phys. 4, 945 (2008).

[47] H. Pichard, A. Duclos, J.-P. Groby, V. Tournat, and V. E. Gusev, Phys. Rev. E 89, 013201 (2014).

[48] E. Bitzek, P. Koskinen, F. Gähler, M. Moseler, and P. Gumbsch, Phys. Rev. Lett. 97, 170201 (2006).

[49] H. Mizuno, K. Saitoh, and L. E. Silbert, Phys. Rev. E 93, 062905 (2016). 